
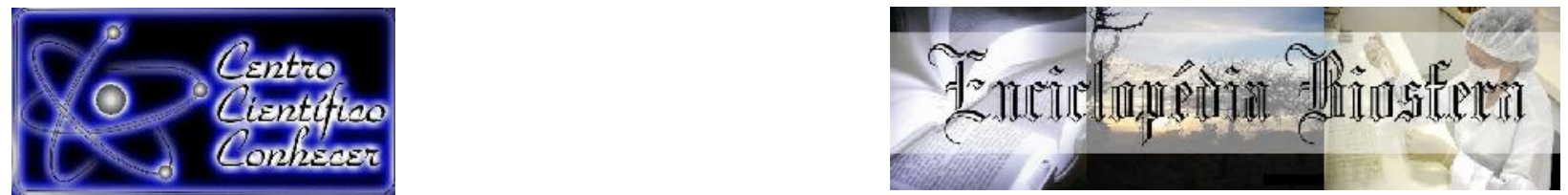

\title{
OCORRÊNCIA DE PARASITAS ZOONÓTICOS EM ANIMAIS DE COMPANHIA NA CIDADE DE BARUERI-SP
}

Victória Guizellini ${ }^{1}$, Isabelle Ronza Pimentel ${ }^{2}$, Márcia Cristina Menão ${ }^{3}$ e Arnaldo Rocha ${ }^{4}$

1- Especialista em Patologia Clínica Veterinária pela Faculdade Qualittas

2- Mestranda no Programa de Saúde Ambiental do Centro Universitário das

Faculdades Metropolitanas Unidas- FMU

3- Professora no Programa de Saúde Ambiental do Centro Universitário das

Faculdades Metropolitanas Unidas- FMU e na Universidade Paulista -UNIP

4- Doutor em Ciências pela Faculdade de Medicina Veterinária e Zootecnia da

Universidade de São Paulo, Veterinário autônomo, e-mail para correspondência: rochaveterinario@hotmail.com

Recebido em: 04/10/2019 - Aprovado em: 30/11/2019 - Publicado em: 15/12/2019 DOI: 10.18677/EnciBio_2019B64

\begin{abstract}
RESUMO
A interação entre cães e seres humanos começou há muito tempo, ainda na préhistória. Estudos apontam benefícios dessa convivência interespecífica, seja pelos reflexos das melhorias da saúde humana, pelo prazer de desfrutar da companhia desses animais ou pela utilização na segurança patrimonial. Porém, esta proximidade aumenta a possibilidade de transmissão de doenças, especialmente as zoonoses. O presente trabalho tem como objetivo demonstrar a ocorrência de parasitas intestinais com potencial zoonótico em cães e gatos da região do município de Barueri, São Paulo, Brasil e discutir as possibilidades de controle destes agentes causadores de doenças. Foram analisadas setenta e quatro amostras fecais empregando-se o método de Faust e Hoffmann. Mais da metade dos animais havia recebido antiparasitários até três meses antes das análises deste estudo e nunca haviam tido suas fezes examinadas anteriormente. Os parasitas mais comumente encontrados foram Giardia sp. em $81 \%$ das amostras, Ancylostoma sp. em 4\% e parasitismo múltiplo por Giardia e Toxocara canis em 4\%, assim como outros parasitas e estruturas como leveduras e Strongyloides stercoralis em $11 \%$ das análises. Considerando a saúde única, que envolve o ambiente, pessoas e animais, e também que os parasitas encontrados eliminam suas formas infectantes nas fezes e que se não destinadas adequadamente contaminam 0 ambiente, conclui-se que há falhas no diagnóstico, tratamento e profilaxia das parasitoses na região estudada evidenciando a necessidade de adequações quanto às orientações médicas veterinárias aos tutores desses animais a fim de mitigar os riscos à saúde de todos os envolvidos.
\end{abstract}

PALAVRAS-CHAVE: Doenças Parasitárias, Saúde Pública Veterinária, Saúde Única. 


\title{
OCCURRENCE OF ZOONOTIC PARASITES IN ANIMALS OF COMPANY IN THE CITY OF BARUERI-SP
}

\begin{abstract}
The interaction between dogs and humans began long time ago in prehistory. Studies show benefits of this interspecific coexistence, whether due to improvements in human health, the pleasure of enjoying the company of these animals or the use of residential security. However, this proximity increases the possibility of disease transmission, especially zoonoses. The present study aims to demonstrate the occurrence of intestinal parasites with zoonotic potential in dogs and cats of the region of Barueri, São Paulo, Brazil and discuss the possibilities of control of these agents that cause diseases. Seventy-four fecal samples were analyzed using the method of Faust and Hoffmann. More than half of the animals had received antiparasitics up to three months prior to the analyzes of this study and had never had their feces examined previously. The most commonly found parasites were Giardia sp. in $81 \%$ of the samples, Ancylostoma sp. in $4 \%$ and multiple parasitism by Giardia and Toxocara canis in 4\%, as well as other parasites and structures like yeasts and Strongyloide stercoralis in $11 \%$ of the analyzes. Considering one health that surrounds the environment, people and animals, and also that the parasites found eliminate their infectious forms in the faeces and that if not destined properly contaminate the environment, it is concluded that there are flaws in the diagnosis, treatment and prophylaxis of the parasitoses in the region studied, evidencing the need for adjustments regarding for the tutors of these animals be adapted in the veterinary medical guidelines in order to mitigate the health risks of all those involved.
\end{abstract}

KEYWORDS: Parasitic Deseases, Veterinary Public Health, One Health.

\section{INTRODUÇÃO}

Parasitas são seres que precisam de outro organismo para sobreviver, desviam nutrientes e na maioria das vezes necessitam de abrigo, numa relação obrigatória, sem a qual esses invasores sucumbem. No parasitismo os benefícios são unilaterais, sempre há prejuízo ao hospedeiro. Estudos relacionados a doenças parasitárias em animais de estimação vêm crescendo pois o elo afetivo e social entre 0 animal e o homem aumentou, tornando ainda mais necessária à compreensão das doenças que acometem os animais, os homens e os problemas de saúde pública decorrentes dessa interação, uma vez que há um aumento na prevalência do parasitismo intestinal, em ambas as partes (STERNEBERG-VAN et al.,2016; OLIVEIRA-ARBEX et al., 2017).

A ocorrência e a transmissão de doenças parasitárias estão ligadas à tríade que envolve o parasita, o hospedeiro e o meio ambiente. As condições encontradas no hospedeiro e no ambiente influenciam diretamente os parasitas, oferecendo condições favoráveis ou desfavoráveis para a sobrevivência e reprodução dos vilões desta história (ROCHA, 2013; DANTAS-TORRES; OTRANTO, 2014).

Existe uma grande variedade de parasitas que dependem da hospedagem e dos nutrientes que só conseguem no lúmen intestinal de cães e gatos, sendo os mais comuns, dentre os protozoários, Giardia sp e Cystoisospora sp e dentre os helmintos Toxocara canis e Ancylostoma sp e Dipylidium caninum. Na espécie 
humana, a Giardia sp pode causar giardíase, o Toxocara canis a Larva Migrans Visceral (LMV), o Ancylostoma caninum a Larva Migrans Cutânea (LMC) e o Dipylidium caninum a dipilidiose (BLAZIUS et al., 2005; FUNADA et al., 2007).

A constatação de parasitoses nos animais de companhia revela a necessidade de empenho dos médicos veterinários, responsáveis pelos diagnósticos, tratamentos e profilaxias dessas zoonoses, que preferencialmente deverão envolver os tutores num processo educativo a fim de obter maior adesão aos esquemas terapêuticos e profiláticos (KATAGIRI; OLIVEIRA-SEQUEIRA, 2007; DI CHIACCHIO, 2013; TORRES-CHABLÉ et al., 2015). Seguido também por uma abordagem "One Health" com o intuito de agregar áreas do conhecimento com a finalidade de imprimir soluções para o impacto dessas doenças, integrando esforços para sua redução com interesse colaborativo englobando práticas de prevenção e vigilância (BAUM et al., 2017; MARDONES et al., 2017).

Tendo em vista as parasitoses que são transmitidas de forma direta, pelo contato com os animais, ou indiretamente, através de fômites, contato com formas infectantes presentes no ambiente ou por vetores, o interesse a despeito dessas zoonoses deve fazer parte das ações dos diversos profissionais da área da saúde, como médicos e médicos veterinários (BANETH et al., 2016).

O objetivo deste trabalho foi determinar a ocorrência de parasitas intestinais em cães e gatos atendidos em clínicas veterinárias na cidade de Barueri, São Paulo, Brasil, de janeiro a abril de 2018, subsidiando o raciocínio epidemiológico para melhoria no diagnóstico, tratamento e medidas preventivas, de promoção da saúde única, inclusive educativas, visando mitigar assim a transmissão desses agentes infecciosos entre os animais e entre os animais e pessoas.

\section{MATERIAL E MÉTODOS}

Foi realizado um estudo prospectivo no período de janeiro a abril de 2018, com um total de 74 amostras fecais analisadas em laboratório de análises clínicas veterinárias situadas no município de Barueri, estado de São Paulo.

A população de cães e gatos foi selecionada entre clínicas veterinárias e tutores de diversos bairros do referido município.

Como fator de inclusão adotou-se: animais encaminhados por veterinários e com requisição para exame complementar de diagnóstico coproparasitológico em que constasse o nome, espécie, raça, idade, origem (canil comercial ou adoção), informações sobre acesso à rua e antiparasitários utilizados em desparasitações prévias.

Para todos os tutores que aceitaram participar da pesquisa foi fornecido um pote coletor universal e foram orientados a colher material fecal recém evacuado, depositar no frasco, identificar e entregar no laboratório imediatamente.

As 74 amostras recebidas para coproparasitológicos foram analisadas quanto ao aspecto de odor, coloração, muco, sangue, alimentos não digeridos e presença de helmintos e protozoários.

Para a pesquisa de parasitas que produzem ovos, oocistos ou trofozoítos leves, utilizou-se o método da centrífugo-flutuação em solução aquosa de sulfato de zinco a 33\%, ou técnica de Faust (FAUST et al. 1938; KATAGIRI; OLIVEIRASEQUEIRA, 2007) e para a pesquisa de ovos pesados ou larvas de helmintos empregou-se a técnica de Hoffman que se baseia na concentração por sedimentação espontânea (HOFFMAN et al. 1934; NOVAES; MARTINS, 2015).

Os resultados foram analisados por estatística descritiva (MANCUSO, 2018). 


\section{RESULTADOS}

No período de 01/01/2018 até 30/04/2018, foram avaliadas 74 amostras fecais de cães e gatos com manifestações clínicas compatíveis com doença parasitária intestinal, todos domiciliados no município de Barueri, São Paulo.

Os resultados obtidos quanto ao aspecto macroscópico foram: $37(50 \%)$ das amostras com consistência firme, $11(15 \%)$ intermediárias, com consistência entre firme e pastosa, 19 (25\%) pastosas, $2(3 \%)$ líquidas e 5 (7\%) mucoides.

Dentre os cães 31 (42\%) não havia sido submetido à vermifugação, 29 (39\%) tinham sido medicados com antiparasitários e de $3(4 \%)$ animais, os proprietários não souberam informar. Considerando os felinos estudados $8(11 \%)$ não havia sido vermifugado, $3(4 \%)$ havia sido vermifugados alguma vez na vida (Figura 1).

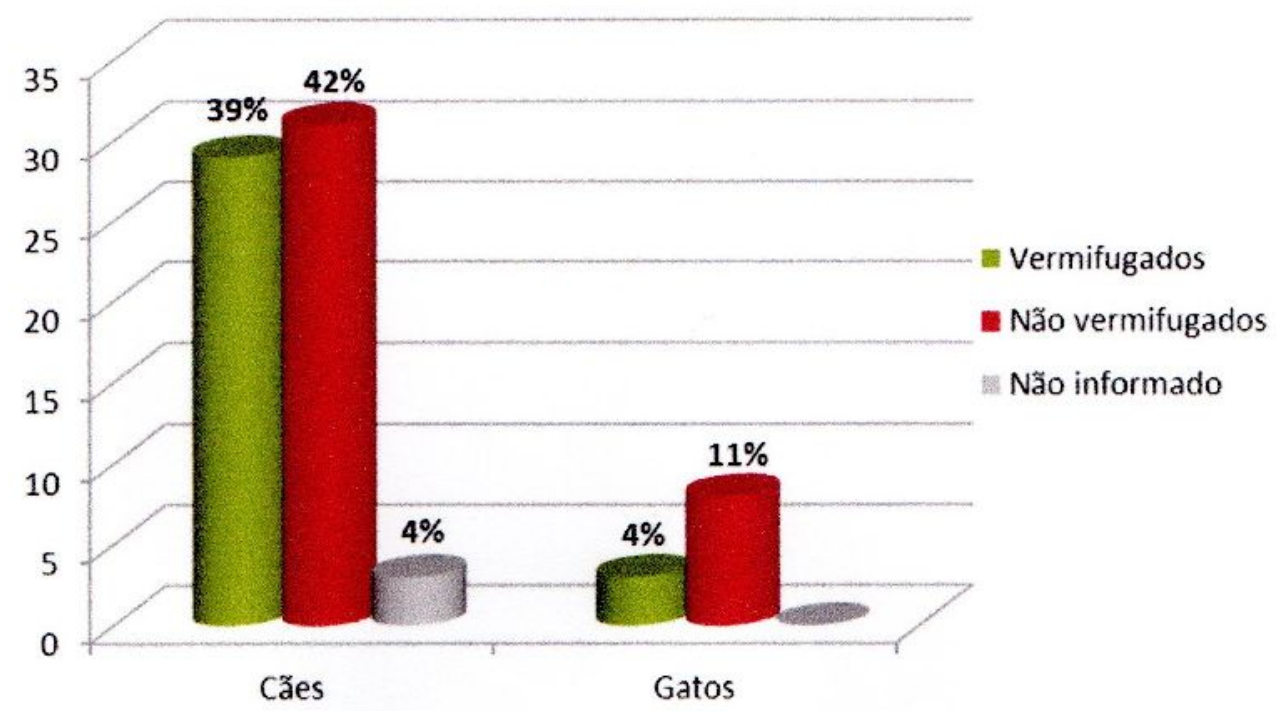

FIGURA 1. Porcentagem de cães e gatos participantes do estudo que receberam antiparasitários antes dos exames coproparasitológicos (Fonte: Autores, 2019).

No presente estudo os parasitas encontrados foram: Giardia sp. 23 (81\%), Strongyloides stercoralis $3(11 \%)$, Ancylostoma sp. 1 (4\%) e parasitismo múltiplo por Giardia e Toxocara canis 1 (4\%) além de leveduras. Das 63 amostras de cães analisadas $21(33,3 \%)$ foram positivas no método de Hoffmann e $42(66,7 \%)$ negativas. Pela técnica de Faust, 5 (8\%) foram positivas e 58 (92\%) negativas.

Considerando-se a espécie felina, das 11 amostras analisadas 5 (45,5\%) foram positivas para Hoffmann e $6(54,5 \%)$ negativas e empregando-se a técnica de Faust, 2 (18\%) foram positivas e $9(82 \%)$ negativas (Figura 2). 


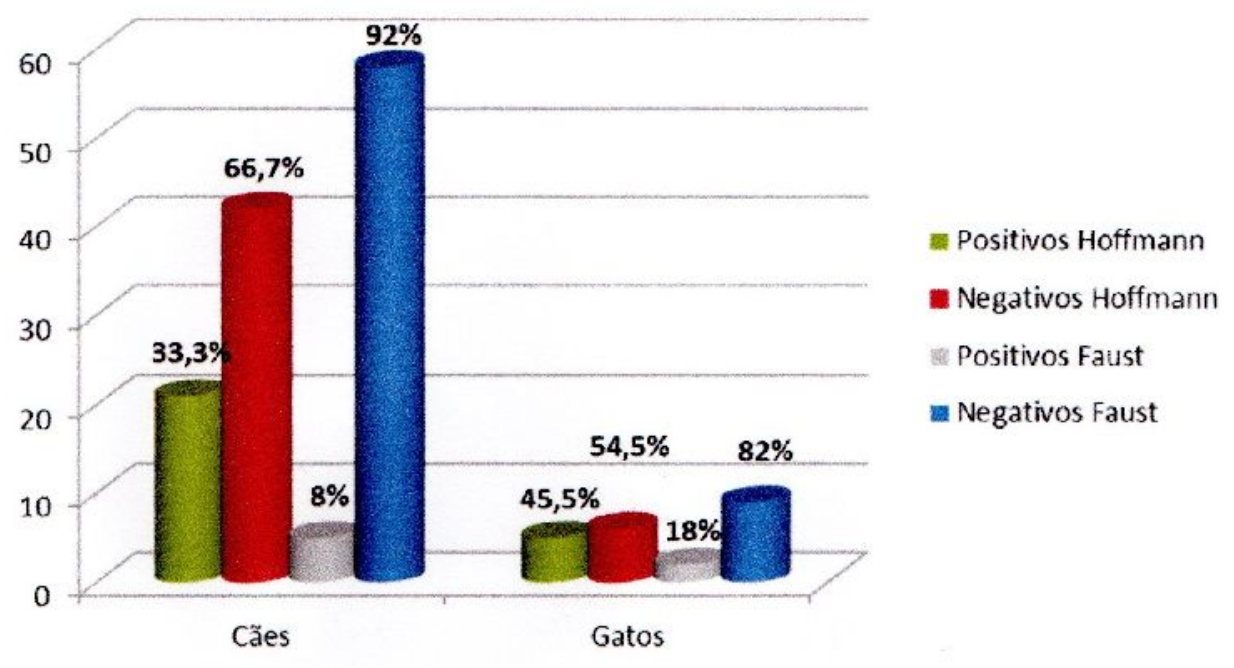

FIGURA 2. Porcentagem de amostras fecais de cães e gatos positivas e negativas para parasitas ao se empregar as técnicas parasitológicas de flutuação e sedimentação. (Fonte: Autores, 2019).

\section{DISCUSSÃO}

As amostras analisadas no trabalho revelaram alta ocorrência de positivos para Giardia sp tanto para cães quanto para gatos, seguido de Ancylostoma sp e Toxocara canis que foram encontrados juntamente com cistos de Giardia, em infecções parasitárias mistas.

Todos os parasitas diagnosticados têm alto potencial zoonótico e os animais positivos oferecem riscos para saúde do tutor pela estreita convivência nas residências. Assim, a limpeza dos locais onde esses animais circulam e evacuam precisa ser realizada frequentemente e com produtos que extingam as formas infectantes desses agentes infecciosos parasitários (ovos, cistos e oocistos).

No sentido de desvendar os diferentes comportamentos biológicos dos parasitas, estudos genéticos têm sido realizados no mundo todo. Nesse sentido, Ortega-Pierres et al. (2017), conduziram estudos moleculares para identificar genes da Giardia que comandam a formação dos cistos e a sensibilidade ou resistência a drogas.

Segundo Quadros et al., (2015), os registros literários recentes demonstram que a giardíase é comumente encontrada em cães errantes e domiciliados, ocorrendo a infecção em animais em condição de superlotação como por exemplo em canis e abrigos, além de animais com imunodepressão.

A maioria dos casos positivos para pelo menos um tipo de parasita em amostras fecais foi diagnosticada em amostras de consistência firme, que é considerada normal para as espécies canina e felina e, sendo assim, a consistência firme não pode servir como parâmetro de ausência de infecção por parasitas intestinais, achados este compatível com outros trabalhos (KATAGIRI; OLIVEIRASEQUEIRA, 2007; FERREIRA et al., 2016).

A presença de Strongyloides stercoralis nas amostras deve ser interpretada com cautela considerando-se uma prevalência subnotificada, essa doença exibe um percalço diagnóstico no que se refere à quantidade insuficiente de larvas excretadas nas fezes, além de que esse helminto pode ser encontrado vivendo livremente no ambiente e se a coleta não foi feita cuidadosamente, pode apresentar contaminação 
ambiental sem, contudo confirmar infecção animal. Sendo indicado o método de Baermann (1917) para o diagnóstico dessa parasitose (ROCHA, 2013; CERVONE et al., 2016).

As duas técnicas parasitológicas escolhidas para comporem as análises neste trabalho são complementares, ou seja, capazes de captar as diversas formas e fases de vida dos diversos parasitas possíveis nas espécies hospedeiras estudadas. Em oposição a resultados verificados na pesquisa de Faust e colaboradores (1938) que revelaram a técnica de centrifugo flutuação em solução aquosa de zinco como mais eficiente para cistos de protozoários, neste trabalho, a técnica que se destacou com maior sensibilidade diagnóstica foi a de Hoffmann, baseada na sedimentação espontânea das formas parasitárias investigadas.

Dentre os animais participantes, haviam domiciliados com acesso livre à rua e outros que saíam guiados por seus tutores em passeios em parques e praças públicas e os gatos por mais que fossem mantidos em espaços controlados, fechados, conseguiam escapar de suas residências para passeios, segundo relatos obtidos em anamnese.

Não foi possível avaliar amostras seriadas de todos os animais, pois uma vez positivos para algum tipo de parasita os veterinários responsáveis pelo envio de novas amostras muitas vezes optaram pelo início imediato do tratamento sem envio de novas amostras para controle.

Comparando o grupo de animais que são mantidos em espaço restrito do domicílio com os que têm acesso a locais públicos constatou-se que os primeiros estavam parasitados enquanto os segundos em sua maioria se revelaram livres de parasitas. Dentre as hipóteses para tal ocorrência, temos a possibilidade de várias exposições a estímulos antigênicos representados por contatos prévios com os parasitas e a resposta imune adequada no caso dos animais com circulação livre, tornando-os imunes a novas e futuras infecções.

Embora todos os tutores revelassem uso de antiparasitário em algum momento da vida dos seus animais de companhia, as incertezas sobre quais produtos, doses, repetições e datas em que foram administrados não permitiram avaliar a eficácia dos princípios ativos e tampouco dos tratamentos realizados. Apesar disto, pode-se notar que a utilização de antiparasitários como vem sendo feita na população estudada não mantém a totalidade dos animais livres de infecções por helmintos e protozoários.

Há de se considerar a dificuldade dos tutores para a percepção de sinais clínicos de doenças, especialmente em felinos, seja porque eles têm manifestações mais discretas ou porque os tutores não são bem treinados para interpretar as alterações físicas ou de comportamento que esses animais apresentam. Assim sendo, os animais que se enquadram como portadores assintomáticos somados à dificuldade que existe em medicá-los resultam num número maior de animais que não são tratados (COELHO et al., 2009).

É fundamental que cães e gatos sejam levados ao veterinário periodicamente, sendo sintomáticos ou assintomáticos, e que o exame coproparasitológico seja realizado em triplicata para aumentar a sensibilidade do teste haja visto que pode haver intermitência na eliminação de ovos, cistos ou oocistos de parasitas nas evacuações (UCHÔA et al., 2017). 


\section{CONCLUSÃO}

Diante da constatação da presença de helmintos e protozoários parasitas intestinais em cães e gatos, com potencial zoonótico, na cidade de Barueri, São Paulo, Brasil, preconiza-se atenção dos médicos veterinários da região e também dos demais profissionais da área da saúde humana e ambiental, reiterando-se a necessidade de diagnóstico, tratamento, prevenção, além de trabalhos educativos de promoção da saúde única concernente às parasitoses intestinais.

\section{REFERÊNCIAS}

BAERMANN, G. Eine einfache Methode zur Auffindung von Ankylostomum (Nematoden) larven in Erdproben. Geneeskundig Tijdschrift voor Nederlandsch Indië, v. 57, p. 131-137, 1917.

BANETH, G.; THAMSBORG, S.M.; OTRANTO, D.; GUILLOT, J.; BLAGA, R.; et al. Major parasitic zoonoses associated with dogs and cats in Europe. Journal of Comparative Pathology. v. 155, p. S54-S74, 2016. Disponível em: <https://www.ncbi.nlm.nih.gov/pubmed/26687277>. doi: 10.1016/j.jcpa.2015.10.179

BAUM, S. E.; MACHALABA, C.; DASZAK, P.; SALERNO, R. H.; KARESH, W. B. Evaluating one health: Are we demonstrating effectiveness? One Health, v.3, p.5-10, 2017. Disponível em: < https: //reader.elsevier.com/reader/sd/pii/S2352771416300143?token=61427BA1E2E5C29 OCAD2182EB3B9BEA0E77A00792652E29C9E17B07FBFD3B66F6B9CBC6137F57 AB05336E88C0CF8BCE3 >. doi: https://doi.org/10.1016/j.onehlt.2016.10.004

BLAZIUS, R.D.; EMERICK, S.; PROPHIRO, J.S.; ROMÃO, P.R.T.; SILVA, O.; Ocorrência de protozoários e helmintos em amostras de fezes de cães errantes da Cidade de Itapema, Santa Catarina. Revista da Sociedade Brasileira de Medicina Tropical, v. 38, p. 73-74, 2005. Disponível em: < http://www.scielo.br/pdf/rsbmt/v38n1/22781.pdf>.doi:http://dx.doi.org/10.1590/S003786822005000100018

CERVONE, M.; GIANNELLI, A.; OTRANTO, D.; PERRUCCI, S. Strongyloides stercoralis hyperinfection in an immunosuppressed dog from France. Revue Vétérinaire Clinique, v. 51, p. 55-59, 2016. Disponível em: < https://www.sciencedirect.com/science/article/pii/S2214567216300011> doi: doi:10.1016/j.anicom.2016.05.001

COELHO, W.M.D.; AMARANTE, A.F.T.; SOUTELLO, R.V.G.; MEIRELLES, M.V.; BRECIANI, K.D.S.; Ocorrência de parasitos gastrintestinais em amostras fecais de felinos no município de Andradina, São Paulo. Revista Brasileira de Parasitologia Veterinária, v. 18, n. 2, p. 46-49, 2009. Disponível em: < http://www.scielo.br/scielo.php?script=sci_arttext\&pid=S1984-29612009000200010>. doi: http://dx.doi.org/10.4322/rbpv.01802010

DANTAS-TORRES, F.; OTRANTO, D. Dogs, cats, parasites, and humans in Brazil: opening the black box. Parasites \& Vectors, v.7, p. 7-22, 2014. Disponível em: <https://www.ncbi.nlm.nih.gov/pubmed/24423244>. doi: 10.1186/1756-3305-7-22 
DI CHIACCHIO, R.G.M.; Ancilostomíase e a Larva Migrans Cutânea. In: ROCHA, A. Parasitologia. São Paulo: Rideel, p. 237-245, 2013.

FAUST, E.C.; D'ANTONI, J.S.; ODOM, V.; MILLER, M.J.; PEREZ, C.; SAWITZ, W.; THOMEN, L.F et al. A critical study of clinical laboratory technics for the diagnosis of protozoan cysts and helminth eggs in feces. I - preliminary communication. American Journal Tropical Medicine Hygiene. v.18, p. 169-183, 1938. Disponível em: $\quad<$ http://www.ajtmh.org/content/journals/10.4269/ajtmh.1938.s1-18.169>. doi: https://doi.org/10.4269/ajtmh.1938.s1-18.169

FERREIRA, J.I.G.S.; PENA, H.F.J.; AZEVEDO, S.S.; LABRUNA, M.B.; GENNARI, S.M.; Occurrences of gastrointestinal parasites in fecal samples from domestic dogs in São Paulo, SP, Brazil. Brazilian journal of veterinary parasitology. v. 25, n. 4, p. 435-440, 2016. Disponível em: < http://www.scielo.br/scielo.php?script=sci_arttext\&pid=S1984-29612016000400435> doi: http://dx.doi.org/10.1590/s1984-29612016081

FUNADA, M.R.; PENA, H.F.J.; SOARES, R.M.; AMAKU, M.; GENNARI, S.M. Frequência de parasitos gastrintestinais em cães e gatos atendidos em hospitalescola veterinário da cidade de São Paulo. Arquivo Brasileiro de Medicina Veterinária e Zootecnia, v. 59, n. 5, p. 1338-1340, 2007. Disponível em:<http://www.scielo.br/scielo.php?script=sci_arttext\&pid=S0102-

09352007000500038>. doi: http://dx.doi.org/10.1590/S0102-09352007000500038

HOFFMAN, W.A.; PONS, J.A.; JANER, J.L. Sedimentation Concentration method in schistosomiasis mansoni. Puerto Rico Journal of Public Health and Tropical Medicine, v. 9, p. 283-298, 1934.

KATAGIRI, S.; OLIVEIRA-SEQUEIRA, T.C.G.; Zoonoses causadas por parasitas intestinais de cães e o problema do diagnóstico. Arquivos do Instituto Biológico, v. $\quad 74, \quad$ n. $2, \quad$ p. 175-184, 2007. Disponível em:< http://www.biologico.sp.gov.br/uploads/docs/arq/v74_2/katagiri.pdf>.

MANCUSO, A. C. B.; CASTRO, S. M. J.; GUIMARÃES, L. S. P. G.; LEOTTI, V. B.; HIRAKATA, V. N.; CAMEY, S. A. Estatística descritiva: perguntas que você sempre quis fazer, mas nunca teve coragem. clinical and Biomedical Research, v. 38, n. 4, p. 414-418, 2018. Disponível em <https://seer.ufrgs.br/index.php/hcpa/article/view/89242>. doi: 10.4322/23579730.89242

MARDONES, F. O.; HERNANDEZ-JOVER, M.; BEREZOWSKI, J. A.; LINDBERG, A.; MAZET, J. A.; et al. Veterinary epidemiology: Forging a path toward one health. Preventive Veterinary Medicine, Part B, v. 137, p. 147-150, 2017. Disponível em: <https://www.ncbi.nlm.nih.gov/pubmed/28081912>.doi:10.1016/j.prevetmed.2016.11. 022

NOVAES, M. T; MARTINS, I. V. F. Avaliação de diferentes técnicas parasitológicas no diagnóstico de helmintoses caninas. Revista Brasileira de Medicina Veterinária, v. 37 , Suplemento 1 , p. 71-76, 2015. Disponível em: < 
http://webcache.googleusercontent.com/search?q=cache:q6kHSgj|GXAJ:rbmv.org/in dex.php/BJVM/article/download/463/350+\&cd=1\&hl=pt-BR\&ct=clnk\&gl=br.

OLIVEIRA-ARBEX, A.P.; DAVID, E.B; OLIVEIRA-SEQUEIRA, T.C.G; KATAGIRI S.; CORADI, S.T.; GUIMARÃES, S. Molecular identification of Ancylostoma species from dogs and an assessment of zoonotic risk in low-income households, São Paulo State, Brazil. Journal of Helminthology, v. 91, n 1, p. 14-19, 2017. Disponível em: <https://www.ncbi.nlm.nih.gov/pubmed/26752269>.doi:10.1017/S0022149X1500114 5

ORTEGA-PIERRES, M.G; JEX, A.R; ANSELL, B.R.E; SVÄRD, S.G. Recent advances in the genomic and molecular biology of Giardia. Acta Tropica v. 184, p. 67-72, 2017. Disponível em: <https://www.ncbi.nlm.nih.gov/pubmed/28888474>. doi: 10.1016/j.actatropica.2017.09.004

QUADROS, R.M.; WEISS, P.H.E.; MILETTI, L.C.; EZEQUIEL, G.W.; MARQUES S.M.T. Ocorrência de Giardia duodenalis em cães domiciliados e apreendidos pelo Centro de Controle de Zoonoses de Lages, Santa Catarina, Brasil. Revista Portuguesa de Ciências Veterinárias. v. 110, p.128-132, 2015. Disponível em: <http://www.fmv.ulisboa.pt/spcv/PDF/pdf12_2015/127-132.pdf>.

ROCHA. A.; Introdução à parasitologia. In: ROCHA, A. Parasitologia. São Paulo: Rideel, p. 1-6, 2013.

STERNEBERG-VAN M.T.; TURNER, D.; VAN T.J., VAARTEN, J. Benefits and risks for people and livestock of keeping companion animals: searching for a healthy balance. Journal of Comparative Pathology, v.155, n.1, p.S8-S17, 2016. Disponível em; https://www.ncbi.nlm.nih.gov/pubmed/26292769 doi: 10.1016/j.jcpa.2015.06.007

TORRES-CHABLÉ, O.M; GARCÍA-HERRERA, R.A; HERNÁNDEZ-HERNÁNDEZ, M.; PERALTA-TORRES, J.A; OJEDA-ROBERTOS, N.F.; et al. Prevalence of gastrointestinal parasites in domestic dogs in Tobasco, south eastern Mexico. Revista Brasileira de Parasitologia Veterinária, v. 24, n. 4, p. 432-437, 2015. Disponível em: < http://www.scielo.br/pdf/rbpv/v24n4/1984-2961-rbpv-S198429612015077.pdf >. doi: http://dx.doi.org/10.1590/S1984-29612015077

UCHÔA, F.F.M.; SUDRÉ, A.P.; MACIEIRA, D.B.; ALMOSNY, N.R.P.; The influence of serial fecal sampling on the diagnosis of giardiasis in humans, dogs, and cats. Revista do Instituto de Medicina Tropical de São Paulo, v. 59, p. 1-6, 2017. Disponível em: <https://www.ncbi.nlm.nih.gov/pubmed/28902297>. doi: $10.1590 / \mathrm{S} 1678-9946201759061$ 